\title{
Inkjet printing as a technique for filling of micro-wells with biocompatible polymers
}

\author{
Marizza, Paolo; Keller, Stephan Sylvest; Boisen, Anja
}

Published in:

Microelectronic Engineering

Link to article, DOI:

10.1016/j.mee.2013.03.168

Publication date:

2013

Document Version

Early version, also known as pre-print

Link back to DTU Orbit

Citation (APA):

Marizza, P., Keller, S. S., \& Boisen, A. (2013). Inkjet printing as a technique for filling of micro-wells with biocompatible polymers. Microelectronic Engineering, 111, 391-395. https://doi.org/10.1016/j.mee.2013.03.168

\section{General rights}

Copyright and moral rights for the publications made accessible in the public portal are retained by the authors and/or other copyright owners and it is a condition of accessing publications that users recognise and abide by the legal requirements associated with these rights.

- Users may download and print one copy of any publication from the public portal for the purpose of private study or research.

- You may not further distribute the material or use it for any profit-making activity or commercial gain

- You may freely distribute the URL identifying the publication in the public portal 


\title{
Inkjet printing as a technique for filling of micro-wells with biocompatible polymers
}

\author{
Paolo Marizza*, Stephan Sylvest Keller, Anja Boisen \\ Department of Micro- and Nanotechnology, Technical University of Denmark, DTU Nanotech, Building 345E, DK 2800 Kongens Lyngby, Denmark
}

\section{A R T I C L E I N F O}

\section{Article history:}

Received 8 October 2012

Received in revised form 20 March 2013

Accepted 26 March 2013

Available online $\mathrm{xxxx}$

\section{Keywords:}

Inkjet printing

Micro-wells

PVP

Drug delivery

\begin{abstract}
A B S T R A C T
We present an innovative technique to dispense precise amounts of polymer solutions into large arrays of microscopic wells. An inkjet printer (NP 2.1 GeSim, Germany) is used to fill micro-wells with poly (vinyl pyrrolidone) (PVP K10). The micro-wells are fabricated with cavity diameters of $300 \mu \mathrm{m}$ down to $50 \mu \mathrm{m}$ with SU-8 with two steps of negative photolithography. Inkjet printing is shown to be a suitable technique to dispense defined volumes of solution (down to $0.3 \mathrm{~nL}$ ) in a highly reproducible way. The filling with polymer can be controlled varying the concentration of the solution and the number of dispensed droplets. Solutions of up to $20 \mathrm{wt}$.\% PVP in water are successfully spotted.
\end{abstract}

(C) 2013 Elsevier B.V. All rights reserved.

\section{Introduction}

Inkjet printing is a familiar technology which has been originally conceived to transfer electronic data onto flexible substrates like paper and overhead transparencies. Recently, a variety of new applications of this technology have been investigated. Versatile tools have been developed to dispense minute amounts of a broad range of materials for various industrial manufacturing processes. Inkjet has been employed for high precision dispensing of polymer into MEMS switches [1], metallic solders in micro-electronic soldering [2], and UV curable epoxy resins for micro-optical applications [3], representing a valuable mold-free manufacturing technique to fabricate large arrays of 3D micro-objects [4]. In more recent years inkjet printing has been introduced in the fields of biotechnology and nanomedicine for large-scale screening of genomic material [5], for cell culturing [6,7], for functionalization of Bio MEMS [8] and for drug delivery applications $[9,10]$. This technique in socalled drop-on-demand mode (DOD) allows dispensing precise volumes of liquid solutions with a quasi-no-waste performance. For this reason it has received increasing attention in the biotechnological and pharmaceutical fields since it enables performing complex analysis on small amounts of material, which are often expensive or in limited supply. A frequent issue in dispensing biological solutions onto flat substrates deals with control of size, shape and relative distance of dots as well as alignment and regularity of realized micro-arrays which occurs when spots are formed with a large number of droplets [11]. As a solution, preliminary micro-patterning of the substrate has been proposed [12]. As alternative approach

\footnotetext{
* Corresponding author. Tel.: +45 45255759.

E-mail address: paom@nanotech.dtu.dk (P. Marizza).
}

for spatial confinement of liquid volumes in nano and pico-liter scale we here present microfabricated SU-8 wells.

SU-8 is a commonly used negative photoresist, well suited for low-cost prototyping of high aspect ratio structures by standard photolithography. Furthermore SU-8 has been shown to exhibit biocompatible properties [13] which makes it a suitable support for cells and biomolecules [14].

Previously, filling of microfabricated wells with discontinuous de-wetting has been demonstrated [15]. The use of inkjet printing for filling of micro-wells having cavity diameter from 50 to $300 \mu \mathrm{m}$ allows to confine liquid volumes of pico up to nanoliter range on precisely arranged positions, preserving the circular shape and the size of dots. To accomplish that we use a commercial system designed to spot on glass slides which prints large arrays of dots with high speed. We dispense micro-arrays of various solutions of PVP. Poly(vinyl pyrrolidone) (PVP) is a biocompatible polymer [16] widely used as excipient for drugs for oral dosage forms [17] as well as promoter of cell growth and differentiation. PVP exhibits high solubility in water and many organic solvents [18], giving low viscous polymer solutions [19].

Additionally, the effect of surface tension on the process of filling the micro-wells is studied by dispensing PVP solutions with different solvents (isopropanol and water). This has shown very different results revealing that the surface tension of the solvent plays a key role in the filling process of three dimensional micro-structures.

\section{Materials and methods}

\subsection{Fabrication of micro-wells}

The fabrication of the micro-wells is a process divided into two main steps. (I) Anisotropic etching is used to define trenches in a 
silicon substrate in order to obtain easy-breakable rectangular substrates fitting into the slots of the inkjet printer tray, (II) negative photolithography is used for the definition of the micro-wells. In Fig. 1 the fabrication steps of the overall process are presented. On a silicon wafer an oxide layer $(2 \mu \mathrm{m})$ is deposited by LPCVD (Fig. 1a). A positive photoresist (AZ 5214e) is spin coated on the front side and patterned by photolithography (Fig. 1b). Afterwards, the same resist is spin coated on the back side. In this way the resist pattern on the front side and the back side layer act as protective mask for the etching of the silicon oxide layer (BHF for $30 \mathrm{~min}$ ) (Fig. 1c). The resist is then stripped and an anisotropic etching in $\mathrm{KOH}\left(4 \mathrm{~h}, 80^{\circ} \mathrm{C}\right)$ defines the trenches for the aligned cleavage of the wafer after micro-well fabrication (Fig. 1d). The remaining oxide layer is then removed in BHF (4 min) to improve SU-8 adhesion in the next fabrication step. For the well fabrication, a first layer of SU-8 2075 (thickness $35 \mu \mathrm{m}$ ) is spin coated and selectively exposed to define the bottom of the micro-wells (Fig. 1e). A second layer $(255 \mu \mathrm{m})$ of the same resist is spin coated and patterned (Fig. 1f). Finally, the uncrosslinked resist is developed in propylene glycol methyl ether acetate (PGMEA) (Fig. 1g). Fig. 2a shows a wafer after microfabrication. The silicon chips are cleaved (Fig. 1h) along the trenches (red lines shown in Fig. 2a) defining a rectangle which fits into the slide slots of the inkjet printer tray (Fig. 2b). Each slide contains ten squares (outlined in green in Fig. 2(a) which contains 625 micro-wells each. The micro-wells are aligned into a $25 \times 25$ matrix. Micro-wells are fabricated with cavity diameters of $50 \mu \mathrm{m}, 100 \mu \mathrm{m}, 200 \mu \mathrm{m}, 300 \mu \mathrm{m}$ corresponding to volumes going from 0.5 to $18 \mathrm{~nL}$. The width of the well walls is $50 \mu \mathrm{m}$ and the center-to-center spacing is $450 \mu \mathrm{m}$ for all wells.

\subsection{Inkjet printing of PVP solutions}

Solution of poly (vinyl pyrrolidone) PVP K10 (Mw $\left.10^{5} \mathrm{Da}\right)$ is dissolved in deionized water and isopropanol in different concentrations (5, 10 and $20 \% \mathrm{wt}$ ). For the more concentrated solutions, viscosity measurements are performed to ensure that the liquid exhibits a Newtonian behavior and a viscosity value below $5 \mathrm{mPa}$. This is the minimum viscosity value for dispensable liquids according to the specifications of the equipment used. All the chemicals are purchased from Sigma-Aldrich.

The inkjet printer used in this work is schematically represented in Fig. 3. It is mainly composed of a XYZ movable inkjet head (Nanoplotter NP 2.1 GeSim, Germany) equipped with a piezo driven dispenser with $70 \mu \mathrm{m}$ orifice (GeSim nanotip) and a slide tray where the substrate is mounted. The solution is aspired by the pipette from a well in a titer plate where the sample is stored at the desired temperature. The inkjet head is monitored by a control unit connected to a computer. A top microscope camera is integrated into the pipette head and allows the alignment of the pipette. The system is supplied with a stroboscope camera (side view camera) to visualize and tune the jet direction and stability, as well as shape and diameter of the generated droplets. The intensity, duration, and frequency of the voltage applied to the piezoelectric actuator of the dispenser are tuned for optimized microwell filling. The optimum set of parameters is a pulsing voltage of $90 \mathrm{~V}$, a pulse width of $90 \mu$ s and a frequency of $175 \mathrm{~Hz}$ for all the solutions. The diameter of the droplets is measured with the stroboscope imaging, and is $47 \mu \mathrm{m}$ for $20 \mathrm{wt} . \%$ PVP and $53 \mu \mathrm{m}$ for 5 and 10 wt.\% PVP.

\subsection{Micro-well filling}

PVP solutions are dispensed by DOD inkjet printing into wells of different sizes. The number of droplets to dispense in each well is estimated dividing the cavity volume by the droplet volume. An overview of the spotting plan is shown in Table 1 . The typical time needed for spotting an array of 625 wells is around $12 \mathrm{~min}$. To increase the polymer content inside the micro-wells, the spotting process is repeated up to three times on the same arrays in subsequent printing sessions, where during each session a solution volume equal to the cavity volume is dispensed into each micro-well. Between the sessions, a waiting time for solvent evaporation is introduced in order to avoid spillover. The evaporation of the drops is observed with the top view camera and the time is measured. The typical drying times for isopropanol and water based-solutions are 1 and 2 min respectively. The polymer concentration dose not
(I) Substrate preparation

(a)

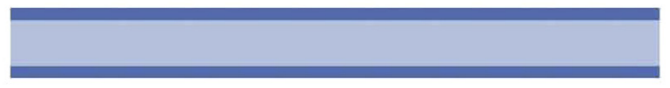

(b)

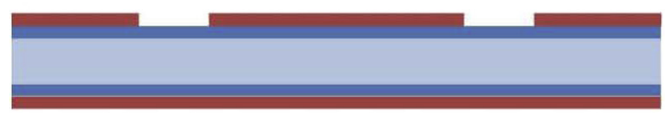

$\longrightarrow$

(c)

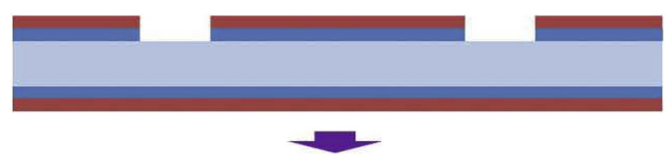

(d)

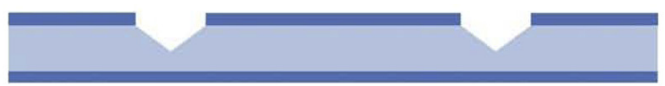

(e)

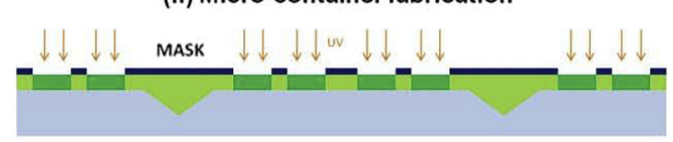

(f)

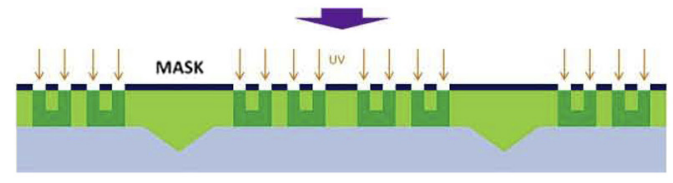

(g)

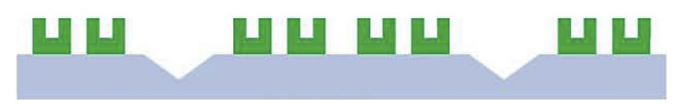

(h)

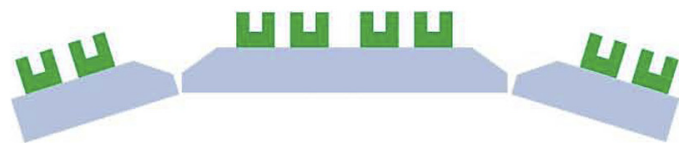

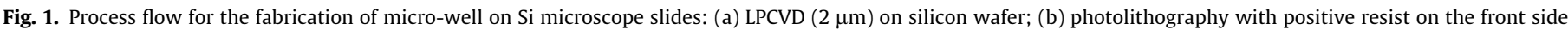

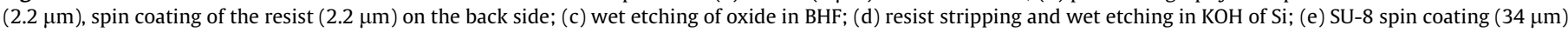
and selective UV exposure; (f) spin coating of second layer of SU-8 (255 $\mu \mathrm{m})$ and masked exposure; (g) SU-8 development; (h) Chips are cleaved in microscope slides. 


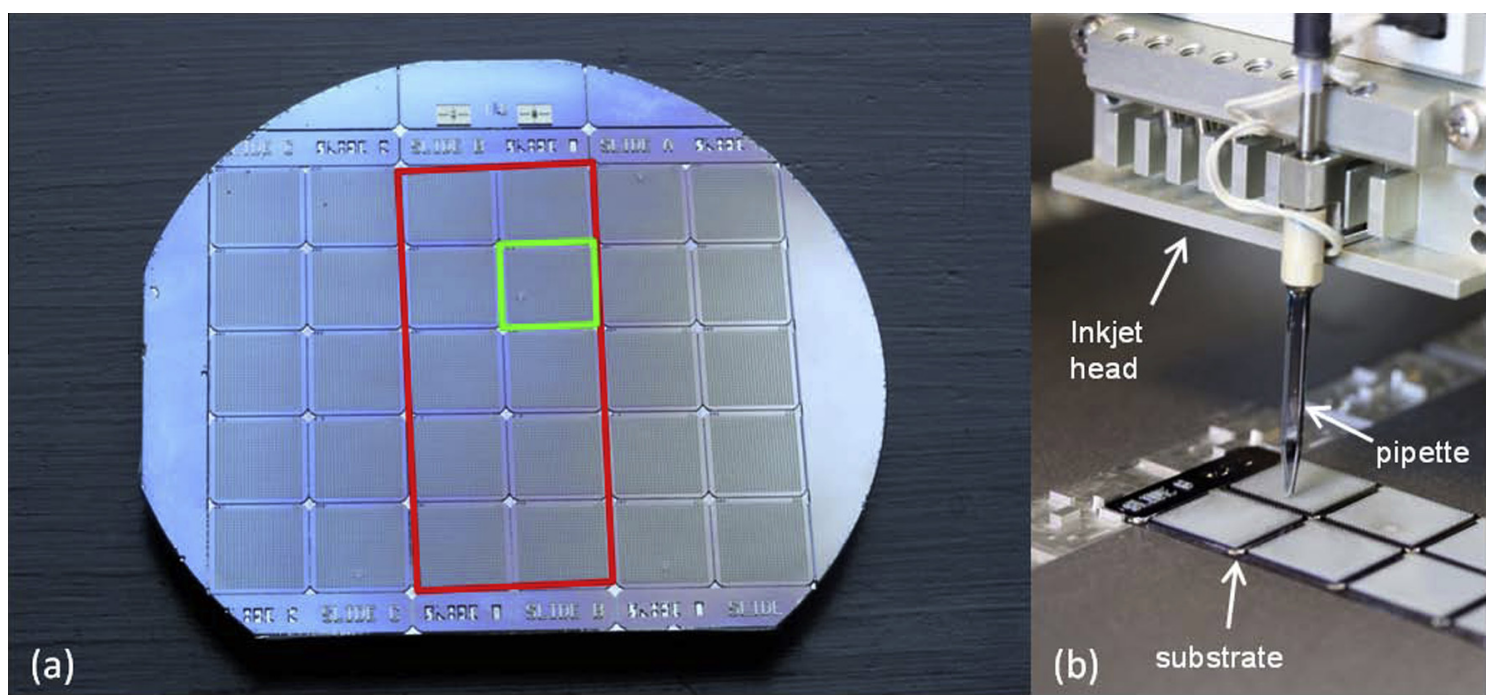

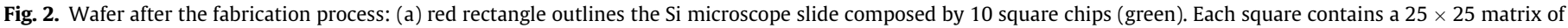

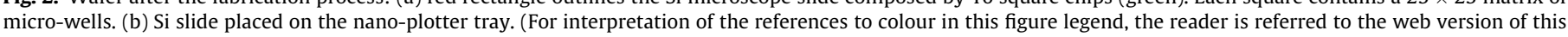
article.)

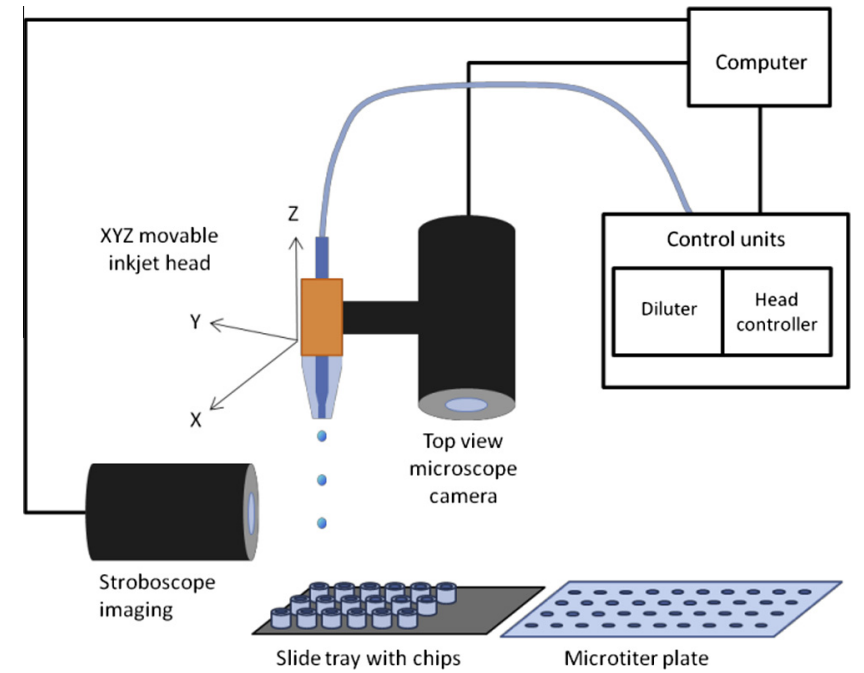

Fig. 3. Scheme of the inkjet printer setup. The micro-pipette is mounted on a XYZ movable head. It aspires the liquid sample to be dispensed from a microtiter plate. The aspiration is driven by a dilutor pump. A functional pipette check takes place in the stroboscope, and if it is successful, the spotting on the substrate begins. During spotting the pressure of the diluter pump is kept constant by system liquid (water).

Table 1

Dispensed volumes of polymer solution for different well sizes for one inkjet session.

\begin{tabular}{llllll}
\hline $\begin{array}{llll}\text { Cavity } \\
\text { diameter } \\
(\mu \mathrm{m})\end{array}$ & $\begin{array}{l}\text { Cavity } \\
\text { volume } \\
(\mathrm{nL})\end{array}$ & \multicolumn{3}{l}{ Dispensed volume for one inkjet session } \\
\cline { 3 - 6 } & & $\begin{array}{l}5 \%, 10 \% \\
\text { wt PVP }\end{array}$ & $\begin{array}{l}\text { Number of } \\
\text { droplets }\end{array}$ & $\begin{array}{l}20 \% \\
\text { wt } \\
\text { PVP }\end{array}$ & $\begin{array}{l}\text { Number of } \\
\text { droplets }\end{array}$ \\
\hline 300 & 18 & 18 & 60 & 18 & 75 \\
200 & 8 & 7.5 & 25 & 7.7 & 32 \\
100 & 2 & 1.8 & 6 & 1.9 & 8 \\
50 & 0.5 & 0.3 & 1 & 0.5 & 2 \\
\hline
\end{tabular}

sensitively affect the evaporation time of dispensed solutions. To measure the filling level of the micro-wells, the chips are coated with an Aluminum layer (15 nm, Alcatel SCM 600). The PVP thickness is then measured by means of an optical profiler (PLu Neox Optical Profiler, Sensofar), equipped with $50 \times$ objective to measure cavity diameter (300 and $200 \mu \mathrm{m})$ and $20 \times(100$ and $50 \mu \mathrm{m})$. The profiler is set in confocal mode, with a monochromatic blue light $(460 \mathrm{~nm})$. Each micro-well is scanned along a cross section passing through the well center within a scanning range of $400 \mu \mathrm{m}$ with a resolution of $1 \mu \mathrm{m}$.

\section{Results and discussion}

\subsection{Level of micro-well filling}

Arrays of wells with cavity diameter of 50, 100, 200 and $300 \mu \mathrm{m}$ are filled with aqueous solutions of PVP at different concentrations. In Fig. 4a a microscope picture of three arrays of filled wells is shown. In Fig. 4b a SEM picture of an empty well (bottom right) and a filled one (top left) is shown. SEM pictures of micro-wells after filling reveal that large arrays of micro-wells can be filled with high precision and a quasi-no-waste deposition (see Fig. 4c) can be achieved when subsequent printing steps are performed on the same wells and a waiting time for solvent evaporation is included. According to the viscosity limit of the dispensing system, a concentration of polymer up to $20 \mathrm{wt}$ \% is successfully spotted. It is observed that water solutions can be spotted with a higher volume than the micro-well cavity, because the high surface tension of water on SU-8 confines the solution in a cap on the top of the micro-structures (see Fig. 5). Upon drying of the liquid, the liquid cap shrinks, and the polymer is deposited in the cavity. After water evaporation the micro-wells are analyzed with scanning electron microscopy and the polymer deposition looks similar to Fig. 4c. The change of jet frequency is also investigated but it did not affect the coating performance. Thus the content of polymer in each micro-well can be controlled by varying its original concentration in the solution and repeating several printing sessions. The level of polymer filling is measured with an optical profiler. An array of 25 micro-wells is scanned for each micro-well dimension, for the three different level of filling (i.e. inkjet sessions), along their cross section passing through the center. In Table 2 the thickness of the polymer layer in the center of the micro-wells is shown. The reported values are given by the difference of the height of the polymer layer and the well bottom thickness $(35 \mu \mathrm{m})$. By comparing 


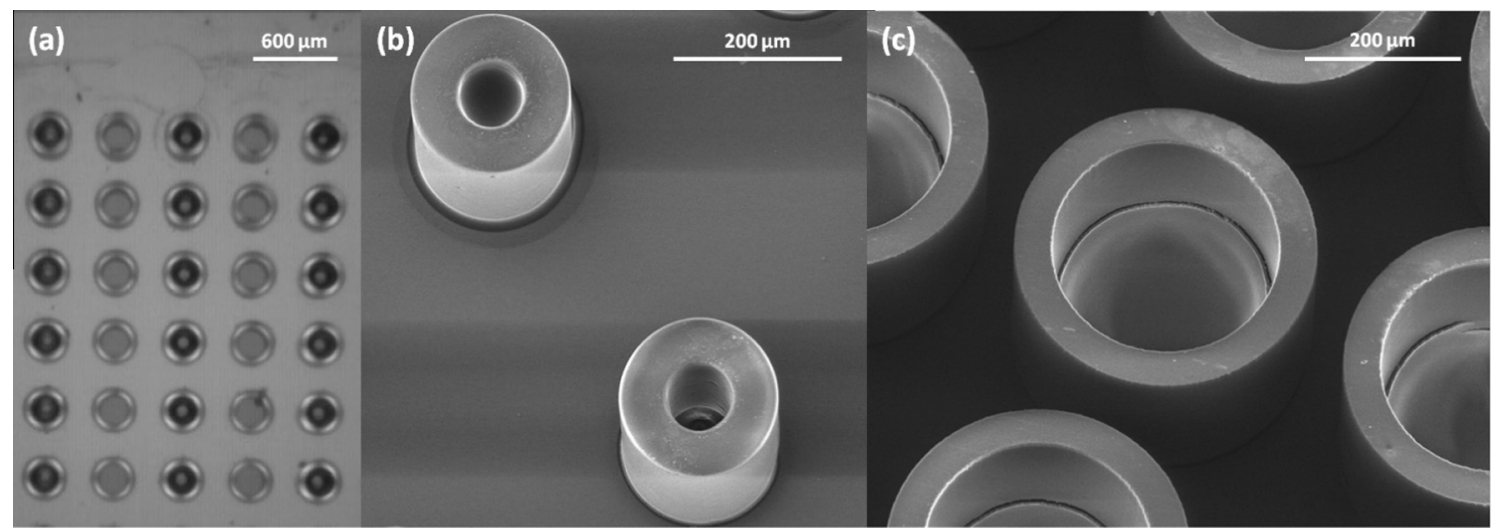

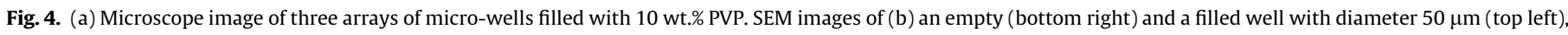
(c) $300 \mu \mathrm{m}$ inner diameter wells filled with $5 \mathrm{wt} . \%$ PVP.

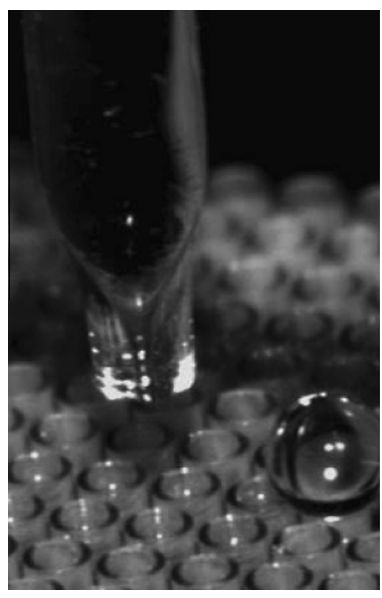

Fig. 5. Microscope picture from the side of one micro-well spotted with 4000 droplets of PVP in deionized water (2 wt\%) with a drop ejection frequency of $100 \mathrm{~Hz}$. the filling level with the corresponding number of droplets it can be noticed that the increase is non-linear. This is due to the fact that the polymer tends to be distributed also on the walls of the wells, and this coating layer gets gradually thicker with the level of filling. The roughness of the surface due to cracks induced by the polymer shrinking does not allow a more accurate computation of the polymer volume by numerical integration.

\subsection{Effect of surface tension on deposition}

The effect of surface tension is investigated by dispensing solutions of PVP in isopropanol. This is motivated by the intention of testing a selective conformal coating of three dimensional microstructures. The deposition of a hydrophilic polymer like PVP can be used to permanently change surface properties of SU-8. For this purpose, solution of 2 wt.\% PVP K10 is dispensed into wells with cavity diameter of $200 \mu \mathrm{m}$. The substrate temperature is kept at $50{ }^{\circ} \mathrm{C}$ in order to shorten the solvent evaporation time. The droplet volume is 0.3 nL. Fig. 6 shows SEM images of wells filled with 1000 droplets at different frequencies: $100 \mathrm{~Hz}$ (Fig. 6a), $200 \mathrm{~Hz}$ (Fig. 6b)

Table 2

Filling level of polymer for different well sizes after one, two and three inkjet sessions with solutions of PVP 20 wt\% in water. Each value is the average of 25 micro-wells.

\begin{tabular}{|c|c|c|c|c|c|c|}
\hline \multirow[t]{2}{*}{ Cavity diameter $(\mu \mathrm{m})$} & \multicolumn{6}{|c|}{ Polymer thickness after subsequent inkjet sessions } \\
\hline & Number of droplets & Polymer height $(\mu \mathrm{m})$ & Number of droplets & Polymer height $(\mu \mathrm{m})$ & Number of droplets & Polymer height $(\mu \mathrm{m})$ \\
\hline 300 & 75 & $8 \pm 2$ & 150 & $20 \pm 1$ & 225 & $24 \pm 1$ \\
\hline 200 & 32 & $88 \pm 7$ & 64 & $132 \pm 9$ & 96 & $155 \pm 2$ \\
\hline 100 & 8 & $102 \pm 3$ & 16 & $223 \pm 7$ & 24 & $246 \pm 5$ \\
\hline 50 & 2 & $125 \pm 5$ & 4 & $182 \pm 4$ & 6 & $282 \pm 8$ \\
\hline
\end{tabular}

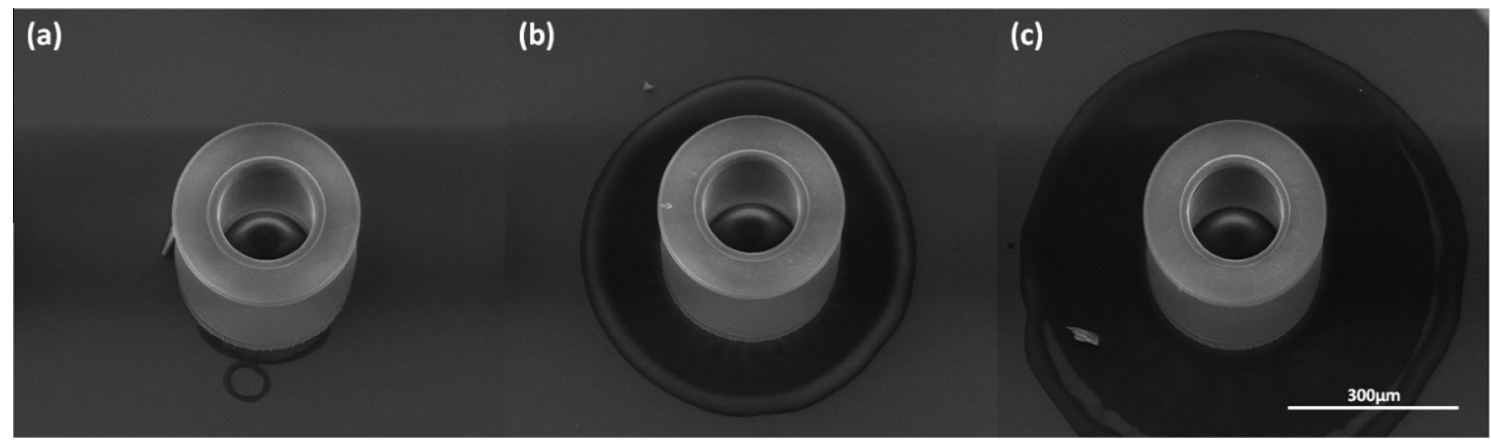

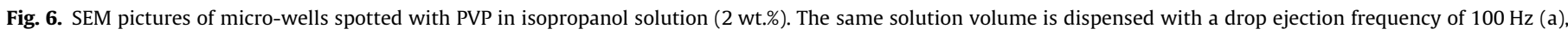
$200 \mathrm{~Hz}(\mathrm{~b})$ and $500 \mathrm{~Hz}(\mathrm{c})$. 


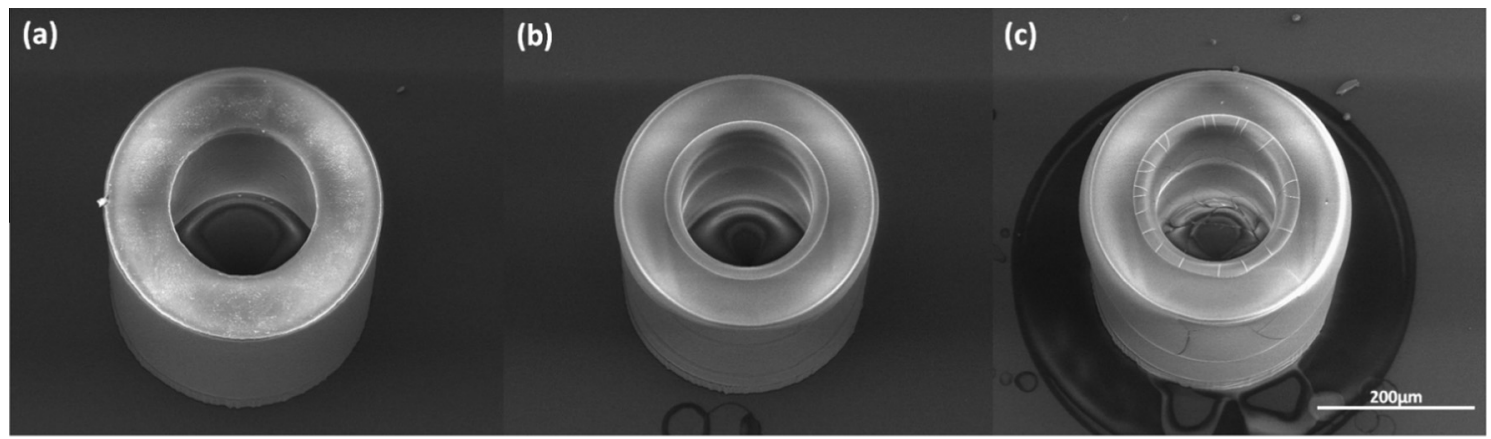

Fig. 7. SEM images of micro-wells filled with an increasing number of droplets: 1000 (a), 2000 (b) and 6000 (c).

and $500 \mathrm{~Hz}$ (Fig. 6c). For all three cases, the same volume of solution is dispensed, with equal droplet size (not depending on the jet frequency), but with different dispensing rates. In any case the solution does not fill the cavity, but tends to wet the surface of the well and covers it with a homogeneous coating. However, at higher frequency, not only the well is coated but also an increasing part of the substrate in between the wells.

In the SEM pictures in Fig. 7 the effect of droplet number on the coating is shown: 1000 (7a), 2000 (7b) and 6000 droplets (7c) are dispensed at a constant frequency of $100 \mathrm{~Hz}$. PVP layer thickness increases with the droplet number. 1000 droplets are dispensed without spillage. These results are explained by the low contact angle of isopropanol on SU-8. Before evaporation, the solvent wets the well distributing the polymer uniformly on its surface. The result is a conformal coating of the three dimensional structure. The effect of frequency can be explained by the different time it takes for the droplets of solution, to dry once they land on the micro-well bottom. The higher the spotting frequency, the longer is the time the solution remains wet. The droplets coalesce and form a liquid mass that symmetrically flows onto the surrounding available surface until the solvent dries.

\section{Conclusions}

In this work we show the fabrication of polymer-filled microwells using DOD inkjet printing technology. By means of wet etching, silicon microscope slides are obtained. On top of them SU-8 micro-wells with cavity volumes down to $0.5 \mathrm{~nL}$ are fabricated with a two step photolithography process. Controlled volumes of PVP in aqueous solutions are dispensed inside the micro-wells, with a good reproducibility in terms of alignment and dispensed volume. Polymer concentrations up to $20 \%$ (wt) are successfully dispensed. Water-based solutions completely fill the cavity volume and the polymer content is controlled by repeated spotting sessions.

Other deposition types are investigated by changing the solvent of the PVP solution. Inkjet printing of isopropanol solutions results in a conformal coating as an effect of the lower contact angle on SU-8. Thus, according to the purpose of the deposition, it is possible to obtain either a conformal coating of the exposed surface or a filling with the same polymer just by changing the solvent of the dispensed solution. The conformal coating with PVP can allow to selectively modify the surface tension of three dimensional micro-structures. This potentially avoids the limitations of other methods, like plasma activation and wet chemical functionalization, which usually change surface properties on wafer scale. In conclusion, we demonstrate a very versatile filling technique for three dimensional micro-structures that can be tuned for several purposes, including promising applications in the field of microdevices for drug delivery and biomedical research.

\section{Acknowledgements}

The authors acknowledge the Villum Kann Rasmussen Foundation for financial support.

\section{References}

[1] S. Nakano, T. Sekitani, T. Yokota, T. Someya, Appl. Phys. Lett. 92 (2008) 053302.

[2] Q. Liu, M. Orme, J. Mater. Process. Technol. 115 (2001) 271-283.

[3] V.J. Cadarso, J. Perera-Núñez, L. Jacot-Descombes, K. Pfeiffer, U. Ostrzinski, A. Voigt, A. Llobera, G. Grützer, J. Brugger, Opt. Express 19 (2011) 18665-18670.

[4] L. Jacot-Descombes, M.R. Gullo, V.J. Cadarso, J. Brugger, J. Micromech. Microeng. 22 (2012) 074012.

[5] T. Goldmann, J.S. Gonzalez, J. Biochem. Biophys. Methods 42 (2000) 105-110.

[6] T. Xu, J. Jin, C. Gregory, J.J. Hickman, T. Boland, Biomaterials 26 (2005) 93-99.

[7] E.A. Roth, T. Xu, M. Das, C. Gregory, J.J. Hickman, T. Boland, Biomaterials 25 (2004) 3707-3715.

[8] P. Cooley, D. Wallace, B. Antohe, J. Assoc. Lab. Autom. 7 (2002) 33-39.

[9] S. Hauschild, U. Lipprandt, A. Rumplecker, U. Borchert, A. Rank, R. Schubert, S. Förster, Small 1 (2005) 1177-1180.

[10] N. Scoutaris, M.R. Alexander, P.R. Gellert, C.J. Roberts, J. Controlled Release 156 (2011) 179-185.

[11] H. Kang, D. Soltman, V. Subramanian, Langmuir 26 (2010) 11568-11573.

[12] J.L. Tan, W. Liu, C.M. Nelson, S. Raghavan, C.S. Chen, Tissue Eng. 10 (2004) 865872.

[13] C. Sung-Hoon, L. Hong Meng, L. Cauller, M.I. Romero-Ortega, L. Jeong-Bong, G.A. Hughes, Sens. J. IEEE 8 (2008) 1830-1836.

[14] G. Voskerician, M.S. Shive, R.S. Shawgo, Biomaterials 24 (2003) 1959-1967.

[15] R.J. Jackman, D.C. Duffy, E. Ostuni, N.D. Willmore, G.M. Whitesides, Anal. Chem. 70 (1998) 2280-2287.

[16] O.Z. Higa, S.O. Rogero, L.D.B. Machado, M.B. Mathor, A.B. Lugão, Radiat. Phys. Chem. 55 (1999) 705-707.

[17] C. Leuner, J. Dressman, Eur. J. Pharm. Biopharm. 50 (2000) 47-60.

[18] V.P. Torchilin, T.S. Levchenko, K.R. Whiteman, A.A. Yaroslavov, A.M. Tsatsakis, A.K. Rizos, E.V. Michailova, M.I. Shtilman, Biomaterials 22 (2001) 3035-3044.

[19] R. Sadeghi, M. Taghi Zafarani-Moattar, J. Chem. Thermodyn. 36 (2004) 665670 . 